University of Nebraska - Lincoln

DigitalCommons@University of Nebraska - Lincoln

$5-2009$

Morph-associated JH titer diel rhythm in Gryllus firmus:

Experimental verification of its circadian basis and cycle characterization in artificially selected lines raised in the field

Anthony J. Zera

University of Nebraska - Lincoln, azera1@unl.edu

Zhangwu Zhao

China Agricultural University, Beijing

Follow this and additional works at: https://digitalcommons.unl.edu/bioscizera

Part of the Microbiology Commons

Zera, Anthony J. and Zhao, Zhangwu, "Morph-associated JH titer diel rhythm in Gryllus firmus:

Experimental verification of its circadian basis and cycle characterization in artificially selected lines raised in the field" (2009). Anthony Zera Publications. 39.

https://digitalcommons.unl.edu/bioscizera/39

This Article is brought to you for free and open access by the Papers in the Biological Sciences at DigitalCommons@University of Nebraska - Lincoln. It has been accepted for inclusion in Anthony Zera Publications by an authorized administrator of DigitalCommons@University of Nebraska - Lincoln. 


\title{
Morph-associated JH titer diel rhythm in Gryllus firmus: Experimental verification of its circadian basis and cycle characterization in artificially selected lines raised in the field
}

\author{
Anthony J. Zera ${ }^{1}$ and Zhangwu Zhao ${ }^{2}$ \\ ${ }^{1}$ School of Biological Sciences, University of Nebraska-Lincoln, Lincoln, NE 68588-0118, USA \\ ${ }^{2}$ College of Agronomy and Biotechnology, China Agricultural University, Beijing 100094, PRC \\ Corresponding author - A. J. Zera, azera1@unlnotes.unl.edu
}

\begin{abstract}
Previous studies demonstrated a high-amplitude, diel cycle for the hemolymph JH titer in the wing-polymorphic cricket, Gryllus firmus. The JH titer rose and fell in the flight-capable morph (long-winged, LW(f)) above and below the relatively temporally invariant JH titer in the flightless (short-winged, SW) morph. The morph-specific JH titer cycle appeared to be primarily driven by a morph-specific diel cycle in the rate of JH biosynthesis. In the present study, cycles of the JH titer and rate of JH biosynthesis in the LW(f) morph persisted in the laboratory under constant darkness with an approximate $24 \mathrm{~h}$ periodicity. The JH titer cycle also shifted in concert with a shift in the onset of the scotophase, was temperature compensated in constant darkness, and became arrhythmic under constant light. These results provide strong support for the circadian basis of the morph-specific diel rhythm of the JH titer and JH biosynthetic rate. Persistence of the JH titer cycle under constant darkness in multiple LW-selected and SW-selected stocks also provides support for the genetic basis of the morph-associated circadian rhythm. The morph-specific JH titer cycle was observed in these stocks raised in the field, in both males and females, in each of 3 years studied. The onset of the cycle in the LW(f) morph, a few hours before sunset, correlated well with the onset of the cycle, a few hours before lights-off, in the laboratory. The morph-specific JH titer cycle is a general feature of G. firmus, under a variety of environmental conditions, and is not an artifact of specific laboratory conditions or specific genetic stocks. It is a powerful experimental model to investigate the mechanisms underlying endocrine circadian rhythms, their evolution, and their impact on life history evolution.
\end{abstract}

Keywords: JH, juvenile hormone, JH biosynthesis, circadian rhythm, biological rhythm, Gryllus, wing polymorphism

\section{Introduction}

Circadian rhythms are ubiquitous and play an important role in coordinating the adaptive expression of various biological traits with the cycle of day and night (Giebultowicz, 2000; Saunders, 2002; Beaver et al., 2002; Hadley and Levine, 2007). In vertebrates, many components of hormonal regulation such as hormone titers, rates of hormone biosynthesis and release, and receptor expression exhibit endogenous circadian cycles (Aschoff, 1979; Nelson, 1995; Kalsbeek et al., 2001). However, the extent to which analogous cycles occur for insect endocrine traits and their functional significance has been much less studied (reviewed in Vafopoulou and Steel, 2005; Steel and Vafopoulou, 2006; see discussion).

Juvenile hormone has long been thought to regulate many key aspects of dispersal polymorphism, most notably wing-polymorphism, in insects (Wigglesworth, 1961; Nijhout, 1994; Zera, 2004; Zera et al., 2007a). The most widely held hypothesis pro- poses that the hemolymph $\mathrm{JH}$ titer below some threshold regulates the expression of developmental and reproductive traits that define the dispersing morph that delays reproduction, while a titer above that threshold produces the alternate suite of traits that characterizes the flightless/reproductive morph (see Section 2 for more detailed description of morph characteristics).

Despite considerable discussion and speculation, only limited published data exist on direct measures of components of JH regulation (e.g. hormone titers) in wing-polymorphic insects (Zera, 2004; Zera et al., 2007a). Moreover, the first direct measurement of the hemolymph JH titer in adult morphs of a wing-polymorphic insect yielded an unexpected finding: a high-amplitude, morph-specific diel cycle for the JH titer in the cricket Gryllus firmus (Zera and Cisper, 2001; Zhao and Zera, 2004a). The JH titer rose and fell about 20-fold near the end of the photophase and beginning of the scotophase on each of days 5-8 of adulthood in the flight-capable morph (long winged, LW(f) with functional flight muscles), and cycled above and below the relatively temporally 
constant titer in the flightless (short winged, SW) morph. The high-amplitude JH titer cycle occurred in each of three LW(f)-selected stocks, but in none of three SW-selected stocks, and thus is a morph-associated genetic polymorphism (Zera and Cisper, 2001). Finally, the morph-specific JH titer diel cycle appeared to be largely, if not exclusively, driven by a morph-specific diel cycle in the rate of JH biosynthesis (Zhao and Zera, 2004b). Thus, although adult $G$. firmus morphs clearly differ dramatically with respect to the hemolymph $\mathrm{JH}$ titer, the difference is due to the presence/absence of a high-amplitude, genetically based, diel titer cycle rather than to a consistently higher titer in one morph compared with the other. Endocrine chronobiology is now an important consideration in functional studies of dispersal polymorphism in insects.

There are numerous unanswered questions regarding the causes, characteristics, and functional significance of the morphspecific JH titer diel cycle in G. firmus (Zhao and Zera, 2004a; Zera et al., 2007b; see discussion). Here we focus on two of these: (1) the extent to which the diel cycle is due to a morph-specific, circadian rhythm, and (2) the extent to which the temporal pattern of $\mathrm{JH}$ titer variation in artificially selected lines measured in the laboratory also occurs under natural field conditions. To investigate the first issue, we characterized the daily profiles of the hemolymph JH titer, and rate of JH biosynthesis, in LW(f) and SWselected lines under experimental conditions that directly test the hypothesis that a diel cycle results from an endogenous circadian rhythm (e.g. persistence under constant darkness, temperature compensation, etc; Giebultowicz, 2000; Saunders, 2002).

With regard to the second issue, the same artificially selected laboratory stocks whose JH titer had been characterized in the laboratory were raised under field conditions in Gainesville, FL, the locality from which founders of the laboratory stocks had been collected. This was done to determine the extent to which the morph-specific JH titer cycle occurs under field conditions that differ in several important respects from the laboratory environment under which the cycle has thus far been exclusively studied (e.g. absolute day length, existence of twilight as opposed to a sharp transition from light to dark, diel change in temperature as well as photoperiod). These field studies were done in concert with a companion study in which the JH titer was measured in morphs of G. firmus and related species directly sampled from natural populations (Zera et al., 2007b). These endocrine-field studies are not only important because they shed light on the characteristics of endocrine daily rhythms in G. firmus under natural conditions, they also provide the most detailed information to date on endocrine traits in the field, an area of insect endocrinology for which very little published data are available (Zera et al., 2007b).

\section{Materials and methods}

\subsection{Morphs of G. firmus}

Like many other wing-polymorphic cricket species, G. firmus consists of a flight-capable morph, with fully developed wings and flight muscles (denoted LW(f)), and a flightless morph with underdeveloped wings and flight muscles (SW) (Walker and Sivinski, 1986; Zera and Denno, 1997; Zera, 2004). The LW(f) morph substantially delays ovarian growth and egg production relative to the SW morph, and thus flight-capability trades off with fecundity, a key feature of wing-polymorphic insects (Johnson, 1969; Harrison, 1980; Zera, 2004). In addition, starting near the end of the first week of adulthood, some LW(f) individuals histolyze their flight muscles and exhibit enhanced ovarian growth that is virtually identical to the SW morph (Zera et al., 1997; Zera and Cisper,
2001; Zera et al., 2007b). This second flightless morph, which is denoted LW(h) (long-winged with histolyzed flight muscles), becomes more common as crickets age (Zera et al., 1997).

\subsection{Stocks and rearing conditions in the laboratory}

All experiments, both in the laboratory and in the field, were conducted on lines of the cricket G. firmus that were artificially selected for the LW(f) or SW morph. The lines were derived from a sample of crickets collected in Gainesville, FL, and are nearly pure-breeding, producing $>90 \%$ of the artificially selected morph. These lines are the same as those that have been used in previous studies of the morph-associated JH titer circadian rhythm in G. firmus (Zera and Cisper, 2001; Zhao and Zera, 2004a, 2004b). Lines are grouped into three blocks, each of which represents an independent selection trial involving a line selected for the LW(f) morph, a line selected for the SW morph, and an unselected control. The number after the line (see below) indicates the block to which it belongs. These lines have been maintained continuously in the laboratory under $16 \mathrm{~h}$ light (L):8 h dark (D) at $28^{\circ} \mathrm{C}$ (see Zera and Cisper, 2001 for additional information on these stocks). All field studies and all laboratory experiments except one (to test for the consistency of persistence of JH titer cycle in multiple genetic stocks) were conducted exclusively on LW and SW lines of Bk-2.

\subsection{Photoperiodic experiments in the laboratory}

Four experiments, involving transfers of crickets from standard rearing conditions $\left(16 \mathrm{~L}: 8 \mathrm{D}, 28^{\circ} \mathrm{C}\right)$ to different photoperiods at the same or different temperatures, were conducted to test the hypothesis that the morph-specific JH titer diel rhythm results from a morph-specific circadian cycle. Just before lights-off on day 3 of adulthood, groups of five males and five females, from the LW(f)-selected and SW-selected lines were placed in plastic boxes $(6$ in. $\times 12$ in. $\times 3$ in.) with each box containing standard diet and a vial of water. Boxes were transferred to one of three new photoperiodic/temperature treatments: constant darkness, $28^{\circ} \mathrm{C}$; constant light, $28^{\circ} \mathrm{C}$; or constant darkness, $20^{\circ} \mathrm{C}$. A single blood sample was subsequently taken from a 6-8-dayold (post-adult eclosion) cricket at a particular time during the photophase or scotophase for JH titer determination. Thus, individuals experienced the new photoperiodic environment for at least three subjective scotophases and two subjective photophases before blood samples were taken. Previous studies (Zhao and Zera, 2004a) demonstrated that the morph-specific JH titer daily rhythm begins on about day 4 and occurs until at least day 8 post-adult eclosion under standard rearing conditions (see Figure 1, top panel). In the present study, hemolymph samples were taken at about 4-6 h intervals with greater clustering of samples before and after the beginning of subjective night in the constant darkness or constant light treatments. This was done because, in the previous study in which crickets were reared under a 16L:8D photoregime, the JH titer changed the most rapidly in the LW(f) morph just before and after the onset of the scotophase (Zhao and Zera, 2004a; see Figure 1). Crickets removed from constant darkness experienced light for about 2-15 min before a blood sample was taken. There is no indication that variation in exposure to light affected the $\mathrm{JH}$ titer or rate of $\mathrm{JH}$ biosynthesis. For example, the last third and first third of samples measured did not differ in $\mathrm{JH}$ titer or rate of $\mathrm{JH}$ biosynthesis.

The rearing protocol used for crickets from which corpora allata were obtained to measure in vitro rate of $\mathrm{JH}$ biosynthesis differed slightly from that described above. In this experiment, females were taken from standard rearing conditions just before 
(A) Reference morph-specific JH titer cycle

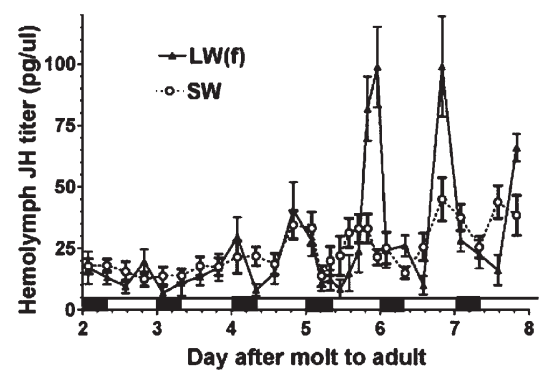

(B) Photoperiod-shift experiment Standard photoperiod (control)

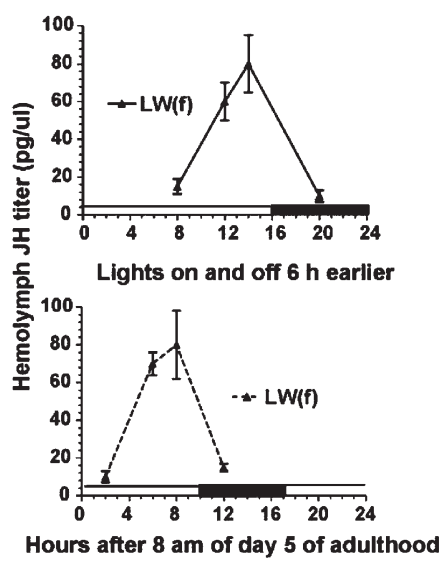

Figure 1. (A) Morph-specific hemolymph JH titer diel cycle in adult, flight-capable (LW(f)) and flightless (SW) female morphs of G. firmus. White bars denote photophase while black bars denote scotophase of standard rearing conditions (16L:8D photoregime, $28^{\circ} \mathrm{C}$ ). Note the high-amplitude JH titer diel cycle in the LW(f) morph but no or only shallow cycle in the SW morph. Data are from Zhao and Zera (2004a). (B) Photoperiod-shift experiment illustrating the correlated shift in the hemolymph JH titer peak in LW(f) G. firmus adult females with a shift in the onset of the scotophase/photophase: (upper panel) JH titer in day $5 \mathrm{LW}(\mathrm{f})$ adult females raised under standard photoperiodic conditions (16L:8D photoregime, $28^{\circ} \mathrm{C}$; lights on at 8am, off at midnight). Bottom panel. JH titer in day 5 adult LW(f) females shifted from the standard photoperiod during the first day of the last stadium to a $16 \mathrm{~L}: 8 \mathrm{D}$ photoregime $\left(28^{\circ} \mathrm{C}\right)$ in which lights were turned on and off $6 \mathrm{~h}$ earlier than in the standard regime. Duration of the last stadium is about 2 weeks. White bars on the $x$-axis denote photophase, while dark bars denote scotophase. Values are means ( \pm S.E.M.); $N=6$ in each case.

lights-off on day 3, placed individually into $16 \mathrm{oz}$ plastic cups containing artificial diet, and transferred into an incubator kept at constant darkness at $28^{\circ} \mathrm{C}$. This modified protocol was used because of the significantly longer period of time required to dissect out the copora allata from individual crickets (5-10 min per individual) compared with the time required to obtain a blood sample. Exposure of crickets reared under constant darkness to light as they awaited dissection of corpora allata was substantially reduced for crickets reared in individual cups. The constant darkness incubator in which the crickets were reared was placed in a room that itself was kept in constant darkness. Food in boxes was changed each day at random with respect to time-of-day to prevent crickets from becoming entrained on time of feeding (Saunders, 2002).

\subsection{Cricket rearing conditions in the field}

To measure the JH titer diel profiles in LW and SW individuals raised under natural photoperiods and temperatures, LW(f)-selected and SW-selected Bk-2 cricket stocks were transported by car to Gainesville, Florida, the location from which founders of the se- lected lines had been collected. Crickets were transported during early September which is just before the period of the time during which dispersal primarily occurs in the field (September to October; Walker, 1986). Crickets were transferred at an age such that nearly all individuals arrived at Gainesville prior to molting into the penultimate stadium (the few penultimate stadium crickets found upon arrival were discarded). Penultimate G. firmus required about 3 weeks to molt into adults in Gainesville and thus experienced the Gainesville photoperiod for more than this period of time prior to blood sampling, since blood samples were taken, at the earliest, from day 5 adults. A phase-shift study conducted prior to transporting the crickets to Florida demonstrated that the $\mathrm{JH}$ titer cycle could be entrained to a new photoperiod in crickets in less than this period of time. LW(f) crickets transferred during the beginning of the last stadium ( 2 weeks before molt to adulthood) to a $16 \mathrm{~L}: 8 \mathrm{D}$ photoregime, in which the onset of darkness (scotophase) had been shifted $6 \mathrm{~h}$ earlier, exhibited a hemolymph $\mathrm{JH}$ titer that was also shifted approximately 6 hours earlier (Figure 1 , middle and bottom panels).

As in the laboratory studies, crickets were reared in the field in 10-gallon aquaria containing cotton-plugged water vials and standard dry diet, at a density of about 80-120 per aquarium during the penultimate and earlier stadia, and about $40-$ 60 per aquarium during the last stadium. Aquaria were placed on a shaded wooden platform adjacent to a field behind the Department of Entomology, University of Florida, Gainesville, FL. Each day, newly molted adults were collected and placed into new aquaria and were kept under the same field conditions until hemolymph samples were taken for JH titer measurement. $\mathrm{Al}$ though the $L W(f)$-selected line primarily produced $L W(\mathrm{f})$ adults, and the SW-selected line primarily produced SW adults, some of the non-selected morph was produced in each line (e.g. SW in the LW-selected stock). As had been done in previous laboratory experiments (Zhao and Zera, 2004a, 2004b), the hemolymph JH titer was only measured in LW(f) individuals from the LW(f)-selected line and SW individuals from the SW-selected line.

On the day of hemolymph sampling, crickets that were to be sampled during the night were placed about 10 per plastic box (6 in. $\times 12$ in. $\times 3$ in.) to reduce the duration of exposure to light of crickets brought into the laboratory while they waited bleeding. As in the previous study of laboratory-reared crickets (Figure 1), most blood samples were taken at $4-6 \mathrm{~h}$ intervals with greater clustering of samples just before or after sunset. Only a single blood sample was taken from an individual cricket.

\subsection{Blood sampling, hormone titer measurements and morph identification}

Hemolymph $(10-20 \mu \mathrm{L})$ was collected into graduated $20 \mu \mathrm{L}$ micropipettes from cuts in cerci, legs, and antennae. As described in more detail (Zera et al., 2007b), hemolymph was blown into $300 \mu \mathrm{L} \mathrm{90 \%} \mathrm{methanol} \mathrm{in} \mathrm{water,} \mathrm{vortexed} \mathrm{for} 1 \mathrm{~min}$ (field) or sonicated for a few seconds (lab), and extracted three times with $600 \mu \mathrm{L}$ hexane. Hexane solutions from an individual were combined in a glass ampule which was flame sealed (field, Gainesville), or were placed in glass vials covered with aluminum foil and parafilm (laboratory, Nebraska). Samples were stored at $-80^{\circ} \mathrm{C}$ (for 1-3 months) until $\mathrm{JH}$ concentration was determined by radioimmunoassay (see below). Samples collected in Gainesville were transported to Lincoln, NE on dry ice. After bleeding, individual crickets were placed in test tubes and frozen at $-20^{\circ} \mathrm{C}$. JH concentration in hexane extracts was measured using a well-validated JH radioimmunoassay (Cisper et al., 2000; Zera and Cisper, 2001; Zhao and Zera, 2004a). In vitro rate of JH biosynthesis was determined using the radiochemical assay of Pratt and Tobe (described in detail in Zhao and Zera, 2004b). 
Flight muscles of LW males and females were examined visually (as in Zera et al., 1997) to determine whether they were large and pink (=functional; defining the LW(f) morph), or whether they were white and reduced (=non-functional; defining the LW(h) morph). Flight muscles of SW adults were also checked to verify that they were white and reduced (non-functional), as has been previously found for all but a few newly molted SW G. firmus (Zera et al., 1997). Ovarian wet mass and whole-body wet mass were also measured for all females.

\subsection{Statistics}

Results of laboratory experiments were not analyzed statistically, since it was obvious by visual inspection of the data whether morphs differed or not in daily rhythms of the $\mathrm{JH}$ titer or rate of $\mathrm{JH}$ biosynthesis. However, results of some field experiments were less clear because of the lower amplitude of the JH titer in the LW(f) morph. Therefore, besides visual inspection of the data, morphspecific differences in the temporal profile of the hemolymph JH titer was assessed by two-way ANOVA, in which morph (LW(f) and SW) and time-of-day were fixed effects. Most importantly, ANOVA was used to test the hypothesis that there was a morph-specific rhythm for the hemolymph JH titer in G. firmus, in which the JH titer changed by a significantly greater degree during a 24-h period in the $L W(f)$ than in the SW morph. Such a pattern of temporal variation should be manifest as a significant MORPH $\times$ TIME-OF-DAY interaction term in the ANOVA. Because there is a specific directionality in the hypothesis being tested (i.e., greater temporal variation in the LW(f) vs. the SW morph) the significance test is onetailed. An analogous test between the LW(f) morph and the other flightless morph, LW(h), was not done because the sample sizes for the $\mathrm{LW}(\mathrm{h})$ morph were often too low for such tests to be undertaken, due to the relative rarity of this morph.
Morph-specific difference in ovarian mass was ascertained by one-way ANOVA of percent body mass due to ovarian mass (arc-sine transformed). Essentially identical results were obtained when untransformed ovarian wet masses were compared.

\section{Results}

\subsection{Laboratory photoperiod experiments}

When shifted from the standard 16L:8D photoregime to constant darkness at the same temperature $\left(28^{\circ} \mathrm{C}\right), \mathrm{LW}(\mathrm{f})$-selected females retained the high-amplitude hemolymph $\mathrm{JH}$ titer cycle, while SW-selected females retained the acyclic JH titer profile (Figure 2). This was the case for $\mathrm{LW}(\mathrm{f})$ and SW females from selected lines of Bk-2 as well as from independently selected Bk1 lines (Figure 2 top and bottom left panels). In both cases, the $\mathrm{JH}$ titer rose in LW(f) females prior to the beginning of the subjective night and decreased dramatically during the subjective night. The titer peak exhibited an approximate $24 \mathrm{~h}$ periodicity. The amplitude of the peaks, and their position relative to the onset of the subjective night, were similar to the amplitude and position of the peaks in these same $\mathrm{Bk}-2 \mathrm{LW}(\mathrm{f})$ and $\mathrm{SW}$ lines raised under standard conditions (16L:8D, $28^{\circ} \mathrm{C}$; Zhao and Zera, 2004a; see Figure 1A).

The JH titer cycle in the long-winged morph also exhibited an approximately $24 \mathrm{~h}$ periodicity, with similar amplitude and position of the peak relative to the onset of the subjective photophase or scotophase, in crickets transferred to constant darkness at $20^{\circ} \mathrm{C}$ or $28^{\circ} \mathrm{C}$ (Figure 2, top left and bottom right panels). Thus, the JH titer cycle was temperature compensated. In contrast to the retention of the JH titer cycle in LW(f) females shifted to constant darkness, the cycle in LW(f) females was lost in crickets transferred to constant light at $28^{\circ} \mathrm{C}$ (Figure 2, top right).
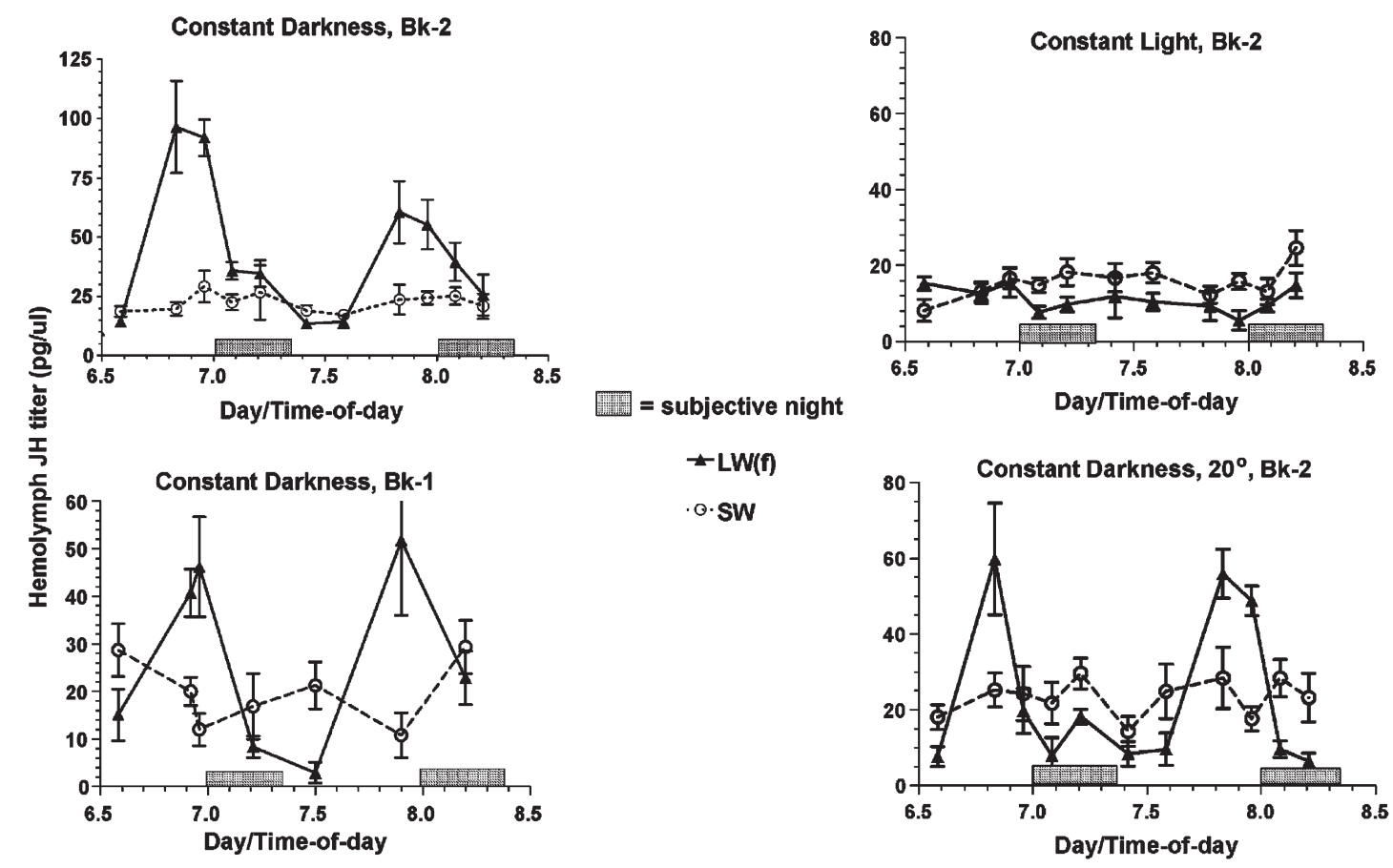

Figure 2. Experiments demonstrating the circadian basis of the morph-specific JH titer diel cycle in G. firmus. Top left panel: retention of the morphspecific JH titer cycle when crickets of Bk-2 LW(f)- and SW-selected lines were transferred from a 16L:8D photoregime to constant darkness. Bottom left: same as top left panel, but for crickets of an independently selected pair of LW(f) and SW lines of G. firmus. Top right: loss of the JH titer cycle in LW(f) crickets that were transferred to constant light. Bottom right: morph-specific JH titer cycle in block-2 LW(f) and SW crickets transferred from 16L:8D to constant darkness but at $20^{\circ} \mathrm{C}$. All other experiments were performed at $28^{\circ}$, the same temperature as standard rearing conditions. Note the similar cycles in this panel and in the top left panel (crickets reared at $28^{\circ} \mathrm{C}$ ), illustrating temperature-compensation of the morph-specific JH titer cycle. Values are means \pm S.E.M. based on sample sizes of 5-8 individual measurements. Gray bars on the $x$-axis denote subjective scotophase. 

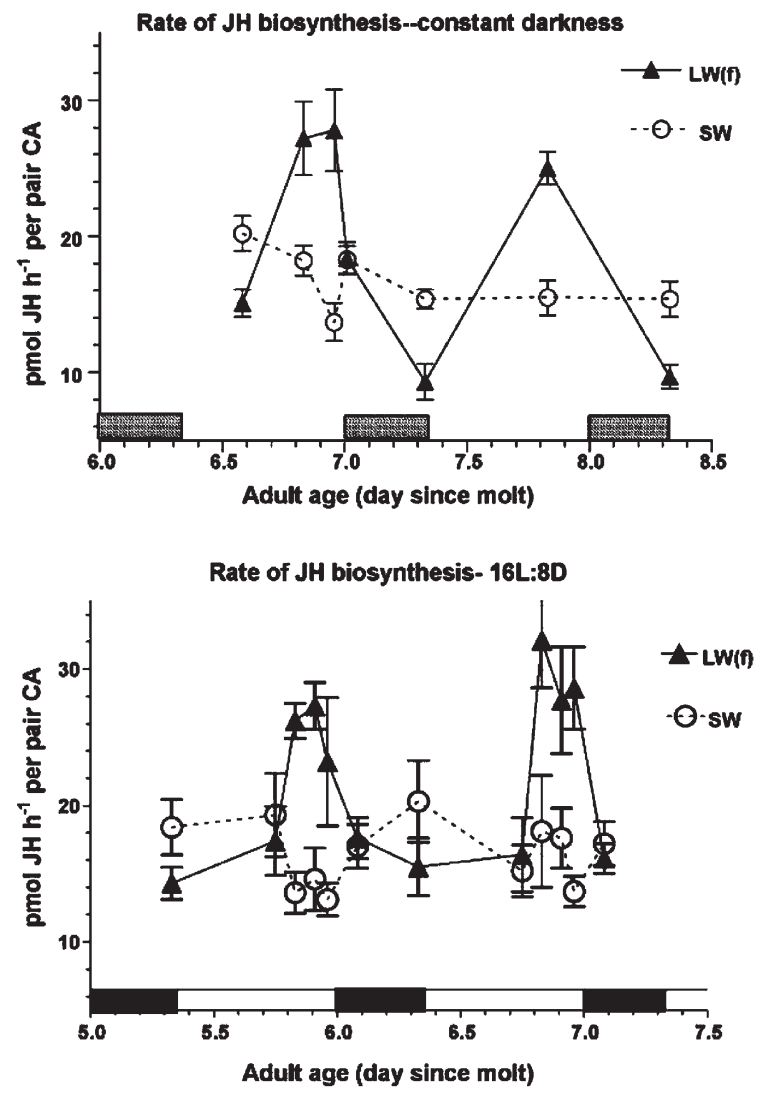

Figure 3. Top panel: retention of the morph-specific daily rhythm for the rate of JH biosynthesis for G. firmus transferred from standard conditions to constant darkness $\left(28^{\circ} \mathrm{C}\right)$. Grey bars on the $x$-axis denote subjective scotophase. Values are means \pm S.E.M. based on 5-6 individual assays. Bottom panel: reference morph-specific rates of JH biosynthesis for LW(f) and SW crickets raised under the standard 16L:8D photoregime, $28^{\circ} \mathrm{C}$ (data from Zhao and Zera, 2004b). Note the similar morphspecific cycles of JH biosynthesis in this panel and the top panel.

As was the case for the hemolymph JH titer, the morph-specific cycle for the rate of $\mathrm{JH}$ biosynthesis, was retained in corpora allata taken from $\mathrm{LW}(\mathrm{f})$ and SW crickets transferred from a 16L:8D photoregime to constant darkness (Figure 3, top panel). The amplitude of the cycle, its approximate $24 \mathrm{~h}$ periodicity, and position of the peak of the cycle relative to the subjective night, in LW(f) females, and absence of a cycle in SW females, all were similar to the previously published, morph-specific cycle of $\mathrm{JH}$ biosynthesis in crickets raised under the standard 16L:8D photoregime (see Figure 3, bottom panel).

\subsection{JH titer and ovarian masses in morphs raised under field conditions}

Standard morph-specific differences in ovarian mass were observed in laboratory-selected lines of G. firmus raised in the field. For example, ovarian mass (as percent of whole-body wet mass) in day 5 females in 2002 was substantially greater in flightless SW females $(19.1 \pm 0.8 \%, N=53)$ or in flightless LW(h) females $(13.5 \pm 1.4 \%, N=15)$ compared with flight-capable $L W(f)$ females $(7.1 \pm 1.0 \%, N=33 ; P<0.001$ in each $t$-test). Similar morph-differences were observed for females of the same or different ages in 2003 and 2004 (data not shown). These morph-differences are similar to those seen for G. firmus stocks measured in the laboratory (e.g. Zera and Cisper, 2001; Zera and Bottsford, 2001) or in females directly sampled from natural populations (Zera et al., 2007b), thus indicating that rearing conditions did not produce unusual morph characteristics in these laboratory-selected lines raised in the field.
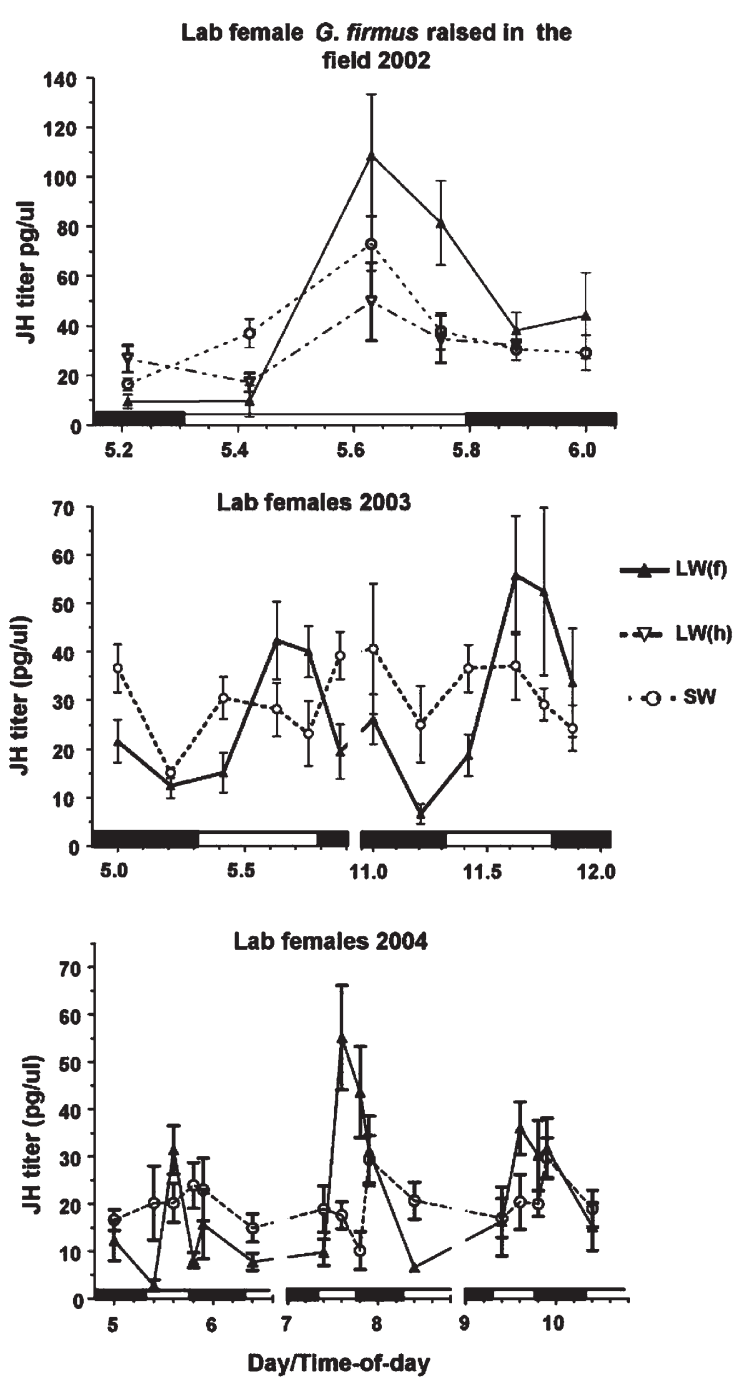

Figure 4. Morph-specific hemolymph JH titer cycle in female crickets of Bk-2 LW(f) and SW-selected lines raised in the field in Gainesville, FL during September to October 2002 (top), 2003 (middle), and 2004 (bottom panels). White bars on the $x$-axis denote day, while dark bars denote night. On the $x$-axis, " $x .0$ " refers to midnight on day " $x$ ". During the sampling period, sunrise occurred between 7:11 and 7:41 a. $\mathrm{m}$. local time ( $x .30-x .32$ on $x$-axis), and sunset occurred between $6: 44$ and 7:44 p.m. local time (0.78-0.81 on $x$-axis). See Section 2 and Zera et al. (2007b) for additional data on field conditions. Note the greater cycling in LW(f) vs. SW females. Values are means \pm S.E.M. Sample sizes in 2002 ranged from 5 to 22 (LW(f) and SW morphs) and from 1 to 7 for the LW(h) morph. In 2003 and 2004, sample sizes ranged from 6 to 10 and 5 to 18 , respectively for $\mathrm{LW}(\mathrm{f})$ and SW morphs.

JH titers in crickets from LW(f)- and SW-selected lines of Bk-2 raised in the field in Gainesville, FL are presented in Figure 4 (females) and Figure 5 (males). These are the same lines that had been characterized previously for the JH-titer profile in females in the laboratory during days 2-8 of adulthood (Zhao and Zera, 2004a; Figure 1A). In day $5 \mathrm{LW}(\mathrm{f})$ adult females, during 2002, there was a 10 -fold increase in the JH titer a few hours before sunset (Figure 4). The JH titer in SW females also exhibited a peak at the same time but the amplitude was much less that seen in the LW morph. Similarly, there was only a slight increase in the JH titer in the other flightless morph, LW(h). The absolute rise in the JH titer peak in field-raised females was similar to that seen in the laboratory females (Zhao and Zera, 2004a; Figure 1A). In 2003, JH titers were again measured on day 5, as well as in older crickets (day 11). On both of these days there was a significant rise in the JH titer in 

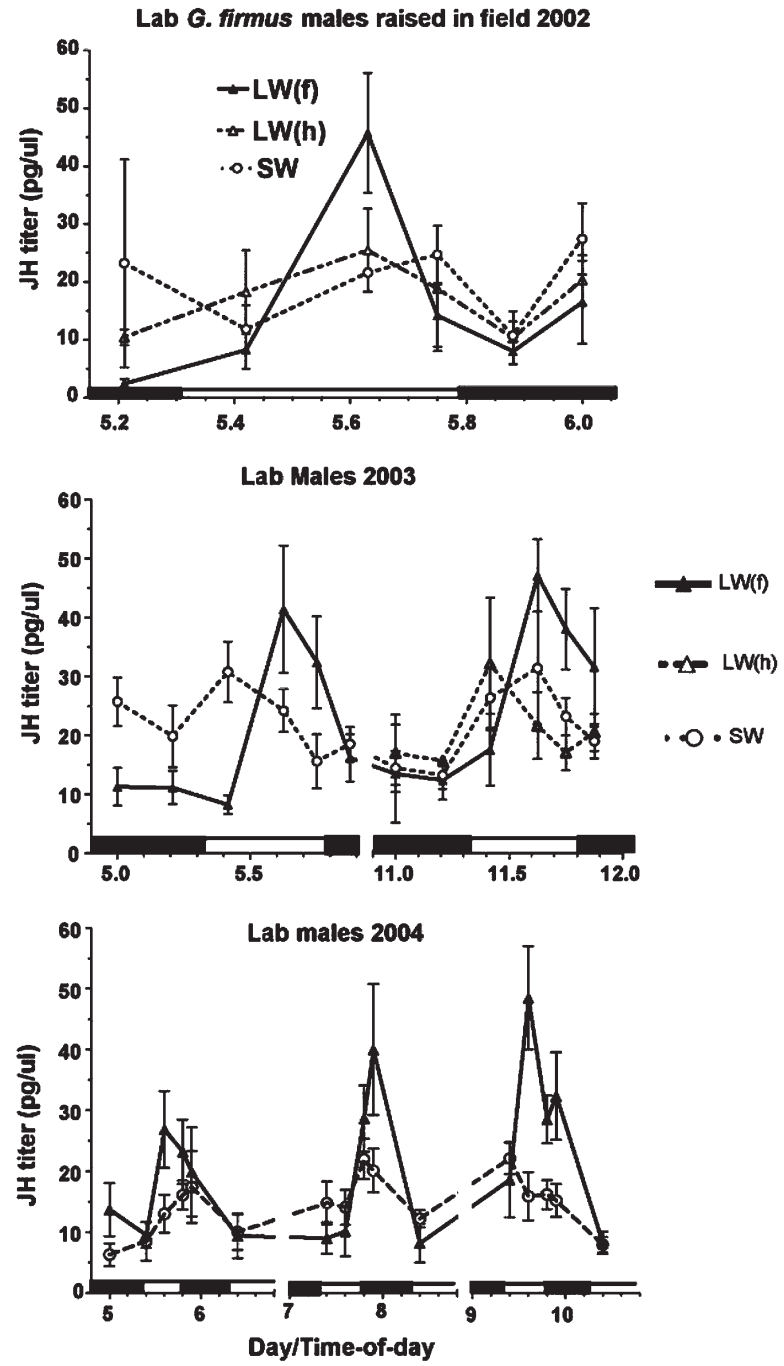

Figure 5. Morph-specific JH titer profiles as in Figure 4, but for male morphs. Means were based on samples of from 6 to 10 individuals for LW(f) and SW morphs and 3 to 5 LW(h) morphs in 2002 (top panel), except for time 5.2 when $2 \mathrm{LW}(\mathrm{f})$ and $3 \mathrm{SW}$ males were sampled, and 5-12 in 2003 and 2004 (middle and lower panels).

LW(f) females, but no, or a much shallower, rise in the flightless SW morph. Absolute titer peaks were lower than in 2002. In 2004, the morph-specific JH titer cycle was observed on each of days 56, 7-8 and 9-10. On each day, the JH titer in the LW(f) morph was lower than that in the SW morph during the early part of the photophase, rose higher than that in the SW female during the latter part of the photophase, and dropped below the SW titer in the following scotophase. Amplitude differences between morphs in the $\mathrm{JH}$ titer on days 7-8 were similar to those in previous years, but $\mathrm{JH}$ titers on days 5-6 and 9-10 differed to a lesser degree between morphs than they did in previous years.

The morph-specific JH titer profiles for males measured in the field were similar to those seen for females. In males during 2002, there was a significant diel cycle with a 10-fold increase in the hemolymph JH titer in the LW(f) morph a few hours before sunset. By contrast, there was no significant diel variation in the JH titer in either the flightless SW morph, or the flightless LW(h) morph. The following year (2003) titers were measured in older (day 11) as well as in day 5 males. In both of these days there was a significant peak in the JH titer in the LW(f) morph at approximately the same time-of-day as in 2002, but no increase, or much less of an increase, in the JH titer in SW or LW(h) morphs. In 2004, titer peaks were observed in the LW(f) morph on each of days 5-6, 78 and 9-10, with titers in the SW morph rising much less or not at all during these times.

In summary, for each age in each year studied in each sex, there was either a significant rise in the JH titer in the $\mathrm{LW}(\mathrm{f})$ morph coupled with no rise in the SW or $\mathrm{LW}(\mathrm{h})$ morphs, or a much greater rise in the flight-capable $\mathrm{LW}(\mathrm{f})$ compared with the flightless SW or LW(h) morphs. Results of statistical analyses confirmed the conclusions derived from visual analysis of the data on the JH titer in field-reared G. firmus. A significant MORPH $\times$ TIME-OF-DAY interaction in the ANOVA of the hemolymph JH titer was observed for most days in females: on day 5, $2002(P<0.05)$; day 5, $2003(P<0.001)$; and day $7(P<0.025)$ and $9(P<0.005), 2004$; but not on day $11(P>0.1), 2003$, or day $5(P>0.2), 2004$. Similarly, a significant MORPH $\times$ TIME-OFDAY interaction for the JH titer in males was observed on day 5 , $2002(P<0.05)$; day $5(P<0.001)$ and $9(P<0.05), 2003$; and day 11 $(P<0.001), 2004$; but not day $5(P<0.1)$ or $9(P<0.2), 2004$.

\section{Discussion}

Endocrine chronobiology is a newly developing area of research in insect endocrinology. In contrast to numerous vertebrate studies, relatively few studies in insects have directly documented daily cycles for various aspects of endocrine regulation, and even fewer have experimentally demonstrated the circadian basis of these cycles, or have investigated functional aspects of the cycles (e.g. Cymborowski et al., 1991; Dai et al., 1995; Ito and Sumi, 1998; for review see Vafopoulou and Steel, 2005). Two notable exceptions are the extensive chronobiological studies of the developmental hormones PTTH (prothoracicotropic hormone) and ecdysteroids in Rhodnius prolixus (reviewed in Vafopoulou and Steel, 2005, and in Steel and Vafopoulou, 2006), and the pheromonotropic neurohormone PBAN in various lepidopterans (Rafaeli et al., 1991; Iglesias et al., 2002; reviewed in Vafopoulou and Steel, 2005).

Natural variation in daily rhythms for endocrine traits, which is particularly important for understanding adaptive evolutionary aspects of hormonal rhythms, has not been studied in detail in either insects or in vertebrates. In addition to the morph-specific JH titer circadian cycle in G. firmus, only a handful of cases of variable endocrine cycles have been reported in insects (Walker and Denlinger, 1980; Das et al., 1993; Ramaswamy et al., 2000; Maxova et al., 2001; Elekonich et al., 2001; Kodrik et al., 2003, 2005), and in only one of these cases (Kodrik et al., 2005) has the circadian basis of the variable diel rhythm been investigated experimentally. Finally, field investigations of endocrine biological rhythms in insects are also rare (Elekonich et al., 2001; Zera et al., $2007 \mathrm{~b}$ ) as, indeed, are field studies of any aspect of insect endocrinology (Zera et al., 2007b).

Previously, we characterized a morph-associated diel rhythm for the hemolymph $\mathrm{JH}$ titer and $\mathrm{JH}$ biosynthetic rate in genetic stocks of G. firmus (Zera and Cisper, 2001; Zhao and Zera, 2004a, 2004b). The strong association between a naturally occurring, genetic polymorphism for a diel endocrine rhythm and an ecologically important genetic polymorphism for dispersal is unique and is a powerful experimental model for investigating many key issues at the interface of chronobiology, endocrinology, and ecology/evolution (discussed below). Important steps in such investigations are verification of the circadian basis of the cycles, and characterization of the morph-specific daily cycle under field conditions.

\subsection{Laboratory studies of the morph-specific circadian rhythm}

Because a daily rhythm can be generated by external factors, and is not necessarily endogenous, the circadian basis of a diel rhythm must be established experimentally (Saunders, 2002). Three types 
of evidence, widely considered to provide strong support for the existence of a circadian rhythm (Giebultowicz, 2000; Saunders, 2002; Vafopoulou and Steel, 2001, 2005), were obtained in the present study. These data convincingly support the hypothesis that the morph-specific diel cycle for the hemolymph JH titer in G. firmus represents an endogenous circadian rhythm. First, the JH titer cycle in the LW(f) morph persisted under constant darkness (i.e., the cycle free-ran; Figure 2). Second, the cycle exhibited an approximately $24 \mathrm{~h}$, period in constant darkness at different temperatures, and thus was temperature compensated (Figure 2). Third, the JH titer cycle was temporally shifted in concert with a shift in the onset of the scotophase (i.e., the cycle was phase-shifted and entrained by light; Figure 1B). Under constant darkness, the amplitude of the JH titer peak in the $\mathrm{LW}(\mathrm{f})$ morph and its position relative to the onset of subjective night were similar to the amplitude and position of the peak relative to the onset of the scotophase in G. firmus raised under the standard 16L:8D photoregime (compare top-left panel of Figure 2 with Figure 1A).

In our previous study (16L:8D photoregime), we observed a low amplitude cycle for the JH titer in the SW morph of G. firmus, in addition to the much higher amplitude JH titer cycle in the LW(f) morph (Zhao and Zera, 2004a; see Figure 1A). In that study, we also found that the hemolymph volume in both the LW(f) and SW morphs contracted by about $25 \%$ from the beginning to the end of the photophase. This suggested that a diel cycle likely exists for total hemolymph volume, which, in turn, causes the lowamplitude JH titer cycle seen in the SW morph (Zhao and Zera, 2004a). Interestingly, we did not observe this low amplitude cycle in the SW morph in any of the three experiments in which the $\mathrm{JH}$ titer was measured under constant darkness (Figure 2). This absence suggests that a diel cycle for total hemolymph volume in $G$. firmus may not be an endogenous rhythm.

The loss of the JH titer cycle in the LW(f) morph under constant light (Figure 2, top right panel), is also consistent with the existence of a circadian rhythm regulated by a photosensitive receptor. Many physiological rhythms persist under constant darkness but dampen and become arrhythmic under constant light (e.g. PTTH, Vafopoulou and Steel, 2001; for reviews see Giebultowicz, 2000; Saunders, 2002). This phenomenon is thought to be caused by the sensitivity of clock gene products, such as the product of the timeless gene, to light.

Persistence of the morph-specific daily cycle in the rate of $\mathrm{JH}$ biosynthesis in G. firmus under constant darkness (Figure 3, top panel), suggests that this trait also is a morph-dependent circadian rhythm. In vitro rate of JH biosynthesis in the cricket Teleogryllus commodus (Ruegg et al., 1986), a species comprised exclusively of long-winged individuals, was the only component of $\mathrm{JH}$ endocrinology that had been explicitly investigated with regard to biological rhythmicity, prior our endocrine studies in G. firmus (Zera and Cisper, 2001; Zhao and Zera, 2004a, 2004b]; present study). Although no evidence for a daily cycle was observed for this trait in T. commodus, sampling was restricted, with the in vitro rate of $\mathrm{JH}$ biosynthesis only being measured over a $24 \mathrm{~h}$ period during a single day of adulthood (day 3 ) in unmated females. The JH titer cycle is barely perceptible in day $3, \mathrm{LW}(\mathrm{f})$ female $G$. firmus (Figure 1A). The rate of JH biosynthesis is also expected to be weak, at best, on day 3, because daily cycles of the JH titer and rate of JH biosynthesis are highly correlated (Zhao and Zera, $2004 b)$. Thus, the existence of a morph-specific cycle in the rate of $\mathrm{JH}$ biosynthesis (and hemolymph JH titer) in T. commodus, similar to that observed in G. firmus, remains an open question.

\subsection{Field studies of the morph-specific rhythm}

Prior to the present study, and the companion study on the JH titer in natural populations of G. firmus (Zera et al., 2007b), pub- lished data on insect endocrine cycles in the field, or indeed on any aspect of insect endocrinology in the field have been very limited (e.g. Elekonich et al., 2001 and references therein; Botens et al., 1997). The most important finding of the field portion of the present study was the existence of a morph-specific JH titer cycle under field conditions that was, in general, similar to the cycle seen in the laboratory. In the field, the JH titer peak in LW(f) females occurred a few hours before sunset, similar to the JH titer peak a few hours before lights-off in the laboratory (compare Figure 4 and Figure 5 with Figure 1A). Also, as observed in the laboratory, the amplitude of the daily cycle of the JH titer was much less in the SW than in the LW(f) morph of field-reared crickets. These patterns were a consistent feature of the morphs raised under field conditions: they were observed in each of 3 years studied, and in each age of female G. firmus studied, except perhaps for day 9 crickets in 2004 when titer peaks did not differ much between LW(f) and SW females.

An analogous cyclic/acyclic JH titer polymorphism also has been reported in the honey bee, Apis mellifera (Elekonich et al., 2001). In this species, the JH titer changed from an acyclic profile in younger worker bees that performed tasks within the hive, to a diel cycle in older foraging workers that flew outside the hive. Thus, in the honeybee, there is a developmental polymorphism with respect to the presence/absence of the JH titer cycle that is associated with a developmental polymorphism for flight-vs. nonflight tasks. The analogous correlation between a JH titer cycle and a phenotype adapted for flight, in both honeybees and G. firmus, suggests a functional relationship between these two traits.

The present study, which measured JH titers in male morphs as well as in female morphs in the field, extends the scope of previous laboratory studies, which only investigated the JH titer in adult female morphs (Zera and Cisper, 2001; Zhao and Zera, 2004a). In each of 3 years, males exhibited a morph-specific JH titer profile that was similar to that observed in females: The hemolymph JH titer peaked a few hours prior to sunset in the LW(f) morph, and the titer in the SW morph was much more temporally constant.

The similar association between the JH titer circadian rhythm and the flight-capable LW(f) morph, in both male and female G. firmus, contrasts with the sex-specific ecdysteroid (ECD) titer difference between morphs of this species (Zera et al., 2007b). In the field, SW females exhibited a consistently higher ECD titer than LW(f) females throughout the day and night, while male morphs did not differ statistically in the ECD titer. It is presently unknown whether the higher ECD titer in SW vs. LW(f) females, is simply the result of a greater release of ECD from the larger ovaries of SW females, without functional consequence, or whether these titer differences regulate some important functional difference between LW(f) and SW females. JH titer differences between morphs of both sexes, likely regulate some sex-independent trait, possibly some aspect of flight which occurs in the $L W(f)$ morph of both sexes.

The general correspondence between the morph differences in the $\mathrm{JH}$ titer cycle observed in the lab and field is important as it indicates that results from laboratory studies can be extrapolated to field conditions and vice versa. This correspondence is useful experimentally because more controlled endocrine studies can be undertaken in the laboratory than in the field. Furthermore, this correspondence also indicates that differences between laboratory and field environments, in such features as presence of twilight, absolute length of the photophase and scotophase, and daily cycle in temperature as well as light/dark do not appear to, at least, strongly influence the expression of the morph-specific JH titer rhythm in G. firmus. Some of these factors, such as twilight as opposed to a direct transition from light to dark are known to influence properties of circadian cycles (Fleissner and Fleissner, 2002). 


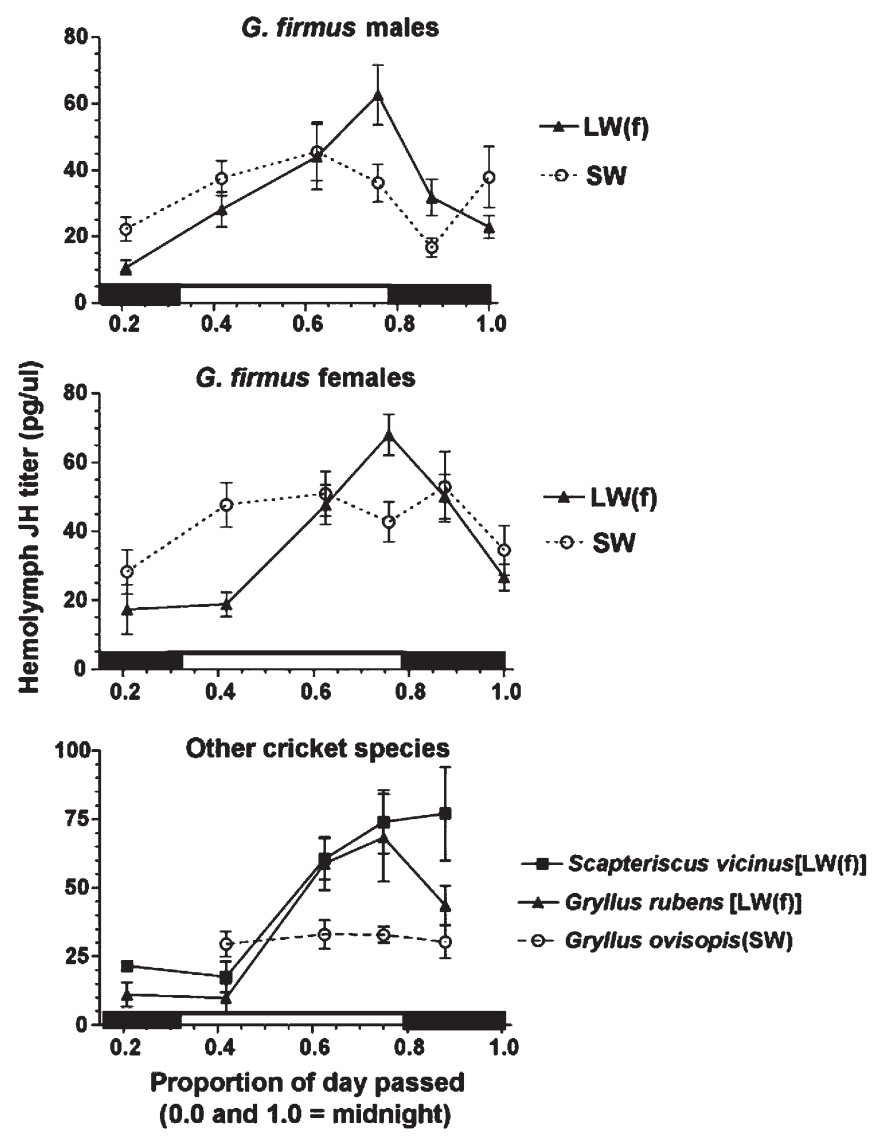

Figure 6. Top and middle panels: morph-specific hemolymph JH titer in field-collected G. firmus males and females. Lower panel: morphspecific hemolymph JH titer profile for other field-collected species of crickets (data in all panels from Zera et al., 2007b). White bars on the $x$-axis denote day, while dark bars denote night. Times of sunrise and sunset are the same as those given in Figure 4.

In the companion study (Zera et al., 2007b), a morph-specific JH titer diel rhythm was also observed in natural populations of G. firmus, in which blood samples were taken from individuals directly collected in the field (summary data of this study are presented in Figure 6). This morph-specific cycle in field-collected LW(f) G. firmus was very similar to that seen in laboratory-selected stocks of G. firmus raised under the same field conditions (compare Figures 4 \& 5 with Figure 6). These data further indicate that the morphspecific endocrine circadian cycle is not an artifact of laboratory selection but rather represents a general feature of laboratory as well as field populations of G. firmus. Moreover, a similar association between a JH titer diel cycle and flight-capability was also seen in several other cricket species (Zera et al., 2007b; Figure 6), indicating that the association observed in G. firmus is not unique to that species, but may be common in crickets.

\subsection{Implications for endocrinology and evolutionary biology}

Identification of a morph-specific $\mathrm{JH}$ titer circadian cycle has many important implications for insect endocrinology and evolutionary biology. For example, this finding indicates that the endocrine regulation of morph-specific traits in wing-polymorphic species is more complex than previously suspected. Clearly, the classic model in which a temporally constant JH titer above or below a single threshold regulates the expression of morph-specific traits is no longer viable, at least for Gryllus.

Zera and Cisper (2001) and Zera et al. (2007b) discuss various more complex models that account for a JH cycle regulating morph-specific differences in flight and reproduction. A key aspect of these models is reconciling the known negative effects of an elevated JH titer on aspects of flight (e.g. histolysis of flight muscles; Zera and Cisper, 2001), with the dramatic rise in the JH titer in LW(f) G. firmus. How can a rise in the JH titer in $L W(f) G$. firmus regulate aspects of flight while not inducing flight-muscle histolysis? There are many possible solutions to this endocrine paradox. Just to give one example, whether an elevated $\mathrm{JH}$ titer has positive or negative effects on aspects of flight might be contingent on the length of time that the JH titer is elevated. Thus, the short-duration rise in the JH titer may be of long enough duration to positively influence aspects of flight or flight-associated traits in a $L W(f)$ individual, while being of insufficient duration to cause negative effects such as flight muscle histolysis, extensive ovarian growth, etc. Other potential endocrine mechanisms that resolve this endocrine paradox are discussed in Zera and Cisper (2001) and in Zera et al. (2007b).

The finding of a morph-specific JH titer circadian rhythm in G. firmus has important practical consequences as well (Zera and Cisper, 2001; Zhao and Zera, 2004a; Zera et al., 2007b). Most importantly, it illustrates the potential for highly erroneous conclusions to be drawn regarding the endocrine regulation of various traits, if such a morph-specific cycle exists but is unknown. For example, depending upon the period of the photophase during which blood samples are obtained, a highly significant positive or negative association between the JH titer and ovarian mass is obtained in G. firmus (Zera and Cisper, 2001). It is conceivable that hormone titers could vary in a morph-specific manner in other types of insect polymorphism, thus warranting investigation of this possibility in any endocrine study of insect polymorphism.

Identification of a morph-specific JH titer cycle in G. firmus also raises fascinating questions, as yet uninvestigated, regarding the proximate mechanisms by which genetic variation in endocrine circadian cycles are produced. Is this morph-specific cycle due to morph-specific differences in aspects of the circadian clock? The output of the clock? Response of target organs, such as the corpora allata, the glands in which JH biosynthesis occurs, to clock output? Another fascinating set of questions relates to the morph-specific traits that are regulated by rhythmic vs. arrhythmic JH titers. Answers to these questions may provide important new insights into basic mechanisms underlying endocrine circadian rhythms and their functional significance.

Finally, endocrine-circadian results reported in the present and earlier studies (Zhao and Zera, 2004b; Zera et al., 2007b) have important evolutionary implications. Studies of life history evolution (e.g. evolution of the timing and amount of reproductive effort; relative duration of the adult and juvenile stages; extent of trade-offs among growth, reproduction and longevity), which are a main focus of evolutionary research, have largely ignored evolutionary aspects of circadian rhythms. Yet an increasing number of life history traits (e.g. various aspects of reproduction) have been shown to exhibit important circadian components (Giebultowicz, 2000; Saunders, 2002; Vafopoulou and Steel, 2005). Similarly, although endocrine studies of life history traits are at the forefront of mechanistic studies of life history evolution (reviewed in Zera et al., 2007a), circadian aspects of endocrine adaptations remain poorly understood. The morph-specific JH titer circadian rhythm that is strongly associated with genetically based wing polymorphism (a life history polymorphism) in G. firmus is a powerful experimental model that provides the opportunity to integrate chronobiology, endocrinology, and life history evolution. Hopefully, such integration will lead to a deeper understanding of the evolution of endocrine circadian rhythms and their role in organismal adaptations. 


\section{Acknowledgments}

This research was supported by NSF grants IOB-0130665 and IOB-0212486 to AJZ.

\section{References}

Aschoff, 1979 J. Aschoff, Circadian rhythms: general features and endocrinological aspects. In: D. Kreiger, Editor, Endocrine Rhythms, Raven Press, New York (1979), pp. 1-63.

Beaver et al., 2002 L. Beaver, B. Gvakharia, T. Vollintine, D. Hege, R. Stanewsky and J. M. Giebultowicz, Loss of circadian clock function decreases reproductive fitness in males of Drosophila melanogaster, Proceedings of the National Academy of Sciences USA 99 (2002), pp. 2134-2139.

Botens et al., 1997 F. W. W. Botens, H. Rembold and A. Dorn, Phaserelated juvenile hormone determinations in field catches and laboratory strains of different Locusta migratoria subspecies. In: S. Kawashima and S. Kikuyama, Editors, Advances in Comparative Endocrinology, Monduzzi, Bologna (1997), pp. 197-203.

Cisper et al., 2000 G. A. Cisper, A. J. Zera and D. W. Borst, Juvenile hormone titer and morph-specific reproduction in the wing-polymorphic cricket, Gryllus firmus, Journal of Insect Physiology 46 (2000), pp. 585-596.

Cymborowski et al., 1991 B. Cymborowski, M. Muszynska-Pytel, P. Porcheron and P. Cassier, Circadian modulation of the ecdysteroid titer in Galleria mellonella larvae, Comparative Biochemistry and Physiology Part A 94 (1991), pp. 431-438.

Dai et al., 1995 J. -D. Dai, A. Mizoguchi, S. Satake, H. Ishizaki and L. I. Gilbert, Developmental changes in the prothoracicotropic hormone content of the Bombyx mori brain-retrocerebral complex and hemolymph: analysis by immunogold electron microscopy, quantitative image analysis, and time-resolved fluorimmunoassay, Developmental Biology 171 (1995), pp. 212-223.

Das et al., 1993 S. Das, O. W. Meier and J. Woodring, Diel rhythms of adipokinetic hormone, fat body response, and haemolymph lipid and sugar levels in the house cricket, Physiological Entomology 18 (1993), pp. 233-239.

Elekonich et al., 2001 M. M. Elekonich, D. L. Schultz, G. Bloch and G. E. Robinson, Juvenile hormone levels in honey bee (Apis mellifera L. ) foragers: foraging experience and diurnal variation, Journal of Insect Physiology 47 (2001), pp. 1119-1125.

Fleissner and Fleissner, 2002 G. Fleissner and G. Fleissner, Perception of natural zeitgeber signals. In: V. Kumas, Editor, Biological Rhythms, Springer-Verlag, Berlin (2002), pp. 83-93.

Giebultowicz, 2000 J. M. Giebultowicz, Molecular mechanism and cellular distribution of insect circadian clocks, Annual Review of Entomology 45 (2000), pp. 769-793.

Hadley and Levine, 2007 M. Hadley and J. Levine, Endocrinology, Pearson Prentice Hall, Upper Saddle River, NJ (2007).

Harrison, $1980-$ R. G. Harrison, Dispersal polymorphisms in insects, Annual Review of Ecology and Systematics 11 (1980), pp. 95-118.

Iglesias et al., 2002 F. Iglesias, P. Marco, M. -C. Francois, F. Camps and E. Fabrias et al., A new member of the PBAN family in Spodoptera litteralis: molecular cloning and immunovisualization in scotophase hemolymph, Insect Biochemistry and Molecular Biology 32 (2002), pp. 901-908.

Ito and Sumi, 1998 M. T. Ito and Y. Sumi, Circadian clock controlling arylalkamine $\mathrm{N}$-acetyltransferase-like activity in the cricket (Gryllus bimaculatus) eggs, Brain Research 799 (1998), pp. 172-175.

Johnson, 1969 - C. G. Johnson, Migration and Dispersal of Insects by Flight, Methuen, London (1969).

Kalsbeek et al., 2001 A. Kalsbeek, E. Fliers, J. Romijn, S. la Fleur, J. Wortel, O. Bakker and E. Endert, The suprachiasmatic nucleus generates the diurnal changes in plasma leptin levels, Endocrinology 142 (2001), pp. 2677-2685.

Kodrik et al., 2003 D. Kodrik, R. Socha and Z. Syrova, Developmental and diel changes of adipokinetic hormone in CNS and haemolymph of the flightless wing-polymorphic bug, Pyrrhocoris apterus (L. ), Journal of Insect Physiology 49 (2003), pp. 53-61.

Kodrik et al., $2005 \rightarrow$ D. Kodrik, R. Socha, S. Zdenka and R. Zemek, The effect of constant darkness on the content of adipokinetic hormone, adipokinetic response and walking behavior in macropterous females of Pyrrhocoris apterus (L. ), Physiological Entomology 30 (2005), pp. 248-255.

Maxova et al., 2001 A. Maxova, D. Kodrik, R. Zemek and R. Socha, Diel changes in adipokinetic response and walking activity of Pyrrhocoris apterus (Heteroptera) in relation to physiological status and wing dimorphism, European Journal of Entomology 98 (2001), pp. 433-438.
Nelson, 1995 R. Nelson, An Introduction to Behavioral Endocrinology, Sinauer Associates, Sunderland, Mass (1995).

Nijhout, 1994 H. F. Nijhout, Insect Hormones, Princeton University Press, Princeton (1994).

Rafaeli et al., 1991 A. Rafaeli, J. Hirsch, V. Soroker, B. Kamensky and A. K. Raina, Spatial and temporal distribution of pheromone biosynthesis-activating neuropeptide in Helicoverpa (Heliothis) armigera using RIA and in vitro bioassay, Archives of Insect Biochemistry and Physiology 18 (1991), pp. 119-129.

Ramaswamy et al., 2000 S. B. Ramaswamy, S. Shengqiang, G. N. Mbata, A. Rachinsky, Y. I. Park, L. Crigler and S. Donald, Role of juvenile hormone-esterase in mating-stimulated egg development in the moth $\mathrm{He}$ liothis virescens, Insect Biochemistry and Molecular Biology 30 (2000), pp. 785-791.

Ruegg et al., $1986 \rightarrow$ R. Ruegg, S. Tobe and W. Loher, Juvenile hormone biosynthesis during egg development in the cricket, Teleogryllus commodus, Journal of Insect Physiology 32 (1986), pp. 517-521.

Saunders, 2002 D. S. Saunders, Insect Clocks, Elsevier, Amsterdam (2002).

Steel and Vafopoulou, 2006 C. G. H. Steel and X. Vafopoulou, Circadian orchestration of developmental hormones in the insect Rhodnius prolixus, Comparative Biochemistry and Physiology Part A 144 (2006), pp. 351-364.

Vafopoulou and Steel, $2001>$ X. Vafopoulou and C. G. H. Steel, Induction of rhythmicity in prothoracicotropic hormone and ecdysteroids in Rhodnius prolixus: roles of photic and neuroendocrine Zeitgebers, Journal of Insect Physiology 47 (2001), pp. 935-941.

Vafopoulou and Steel, $2005 \rightarrow$ X. Vafopoulou and C. G. H. Steel, Circadian organization of the endocrine system. In: L. I. Gilbert, I. Kostas and S. S. Gill, Editors, Comprehensive Molecular Insect Science, Elsevier, Amsterdam (2005), pp. 551-650.

Walker and Denlinger, 1980 - G. P. Walker and D. L. Denlinger, Juvenile hormone and moulting hormone titres in diapause and non-diapause destined flesh flies, Journal of Insect Physiology 26 (1980), pp. 661-664.

Walker, 1986 T. J. Walker, Monitoring the flights of field crickets (Gryllus app. ) and tachinid fly (Euphasiopteryx ochracea) in northern Florida, Florida Entomologist 69 (1986), pp. 678-685.

Walker and Sivinski, 1986 T. J. Walker and J. M. Sivinski, Wing dimorphism in field crickets (Orthoptera: Gryllidae: Gryllus), Annals of the Entomological Society of America 79 (1986), pp. 84-90.

Wigglesworth, $1961>$ V. B. Wigglesworth, Insect polymorphism - a tentative synthesis. In: J. S. Kennedy, Editor, Insect Polymorphism, Royal Entomological Society, London (1961), pp. 103-113.

Zera, 2004 A. J. Zera, The endocrine regulation of wing polymorphism: state of the art, recent surprises, and future directions, Integrative and Comparative Biology 43 (2004), pp. 607-616.

Zera and Bottsford, 2001 A. J. Zera and J. Bottsford, The endocrinegenetic basis of life-history variation: relationship between the ecdysteroid titer and morph-specific reproduction in the wing-polymorphic cricket, Gryllus firmus, Evolution 55 (2001), pp. 538-549.

Zera and Cisper, 2001 A. J. Zera and G. A. Cisper, Genetic and diurnal variation in the juvenile hormone titer in a wing-polymorphic cricket Implications for the evolution of life histories and dispersal, Physiological and Biochemical Zoology 74 (2001), pp. 293-306.

Zera and Denno, 1997 A. J. Zera and R. F. Denno, Physiology and ecology of dispersal polymorphism in insects, Annual Review of Entomology 42 (1997), pp. 207-231.

Zera et al., 2007a $>$ A. J. Zera, L. G. Harshman and T. Williams, Evolutionary Endocrinology: The developing synthesis between endocrinology and evolutionary genetics, Annual Review of Ecology, Evolution and Systematics 38 (2007), pp. 793-817.

Zera et al., 1997 A. J. Zera, J. Sall and K. Grudzinski, Flight-muscle polymorphism in the cricket Gryllus firmus: muscle characteristics and their influence on the evolution of flightlessness, Physiological Zoology 70 (1997), pp. 519-529.

Zera et al., 2007b $>$ A. J. Zera, Z. Zhao and K. Kaliseck, Hormones in the field: evolutionary endocrinology of juvenile hormone and ecdysteroids in field populations of the wing-dimorphic cricket Gryllus firmus, Physiological and Biochemical Zoology 80 (2007), pp. 592-606.

Zhao and Zera, 2004a Z. Zhao and A. J. Zera, The hemolymph JH titer exhibits a large-amplitude, morph-dependent, diurnal cycle in the wing-polymorphic cricket, Gryllus firmus, Journal of Insect Physiology 50 (2004), pp. 93-102.

Zhao and Zera, 2004b Z. Zhao and A. J. Zera, A morph-specific daily cycle in the rate of JH biosynthesis underlies a morph-specific daily cycle in the hemolymph JH titer in a wing-polymorphic cricket, Journal of Insect Physiology 50 (2004), pp. 965-973. 